\title{
Giant breast hamartoma in a 41-year-old female: A case report and literature review
}

\author{
ZHI WANG ${ }^{1,2}$ and JIANJUN HE ${ }^{3}$ \\ ${ }^{1}$ Department of Oncology, Xi'an Jiaotong University College of Medicine, Xi'an, Shaanxi 710049; ${ }^{2}$ Department of Surgery, \\ Tuberculosis Hospital of Shaanxi Province, Xi'an, Shaanxi 710100; ${ }^{3}$ Department of Oncology Surgery, \\ First Affiliated Hospital of Xi'an Jiaotong University, Xi'an, Shaanxi 710061, P.R. China
}

Received October 23, 2014; Accepted August 17, 2015

DOI: $10.3892 / \mathrm{ol} .2015 .3794$

\begin{abstract}
Breast hamartoma is an uncommonly reported benign breast lesion of uncertain cause and pathogenesis. The diagnosis of breast hamartoma by a single method such as mammography, magnetic resonance imaging or sonography is inadequate. In the majority of cases, the breast hamartoma is excised a few years after it has occurred when it is not too big. In the present report, however, a particularly large lesion with a long history is described. Such a case has rarely been reported and shows the necessity of early surgery to reduce trauma as much as possible. Excision of hamartoma was successfully performed and an $11 \times 9 \times 3.5-\mathrm{cm}$ tumor was completely excised. The present study also reviews the literature on breast hamartoma.
\end{abstract}

\section{Introduction}

Breast hamartoma is an uncommonly reported benign breast lesion of uncertain cause and pathogenesis. The majority of affected patients are $>35$ years old, with a mean age of $\sim 45$, and the tumor exhibits a female predominance. Of all the benign breast tumors in women (1-3), only $0.1-0.7 \%$ are diagnosed as breast harmartomas. The diagnosis of breast hamartoma by a single method such as mammography, magnetic resonance imaging or sonography is inadequate. The tumor is often misdiagnosed as fibroadenoma, even upon fine-needle aspiration (FNA). Therefore, the actual incidence could be higher than that reported (4). This benign lesion grows slowly and could become bigger if no intervention is given, which results in greater trauma for the patient (5). In the majority of cases, the breast hamartoma is excised a few years after it has occurred, when it is not too big. In the present study, however, a particularly large lesion with a long history is described.

Correspondence to: Professor Jianjun He, Department of Oncology Surgery, First Affiliated Hospital of Xi'an Jiaotong University, 277 Yantaxi Road, Xi'an, Shaanxi 710061, P.R. China E-mail: chinahjj@163.com

Key words: adipose, breast hamartoma, pseudoangiomatous stroma
Such a case has rarely been reported and shows the necessity of early surgery. The study also presents a literature review on breast hamartoma. Written informed consent was provided by the patient for the publication of this case report and the accompanying images.

\section{Case report}

A 41-year-old female with a 10-year-history of a painless lump in the right breast presented to the First Affiliated Hospital of Xi'an Jiaotong University (Shaanxi, China) in March 2014. The mass had been gradually increasing in size over this period. The patient complained of significant pain in the right breast during menstruation, which was relieved at its conclusion. The risk of breast cancer was not increased, and the patient did not have any of the familial factors. No history of ipsilateral breast trauma, surgery or radiation exposure was reported. No nipple discharge was apparent and no pyrexia was found in the patient's case history.

Upon physical examination, an oval, palpable but painless, smooth, rubbery mass of $\sim 12 \times 10 \mathrm{~cm}$ in size was found in the upper outer quadrant of the right breast. The mass had neither skin fixation nor fixation to adjacent structures in the breast, and its most posterior margin could be defined. No skin ulceration was found. The results of routine laboratory investigations were within normal limits.

Mammograms showed a well-circumscribed mass lesion of mixed density in the right breast. No ipsilateral axillary nodes were detected. The serum level of cancer antigen 15-3 remained normal. Ultrasound of the right breast revealed a solid heterogeneous mass of $10 \times 11 \mathrm{~cm}$. The patient refused to undergo FNA cytology examination.

In order to achieve diagnostic and therapeutic goals, a surgical treatment was planned. Excision of hamartoma was successfully performed and a tumor of $11 \times 9 \times 3.5 \mathrm{~cm}$ was completely excised (Fig. 1). Gross examination revealed an oval, white-reddish, well-defined, encapsulated nodule with a smooth margin. The nodule was soft and possessed a fibrotic envelope at the time of surgery (Fig. 2). The cut surface was smooth and exhibited a grey-reddish color. Adipocytic areas within a fibrotic stroma could be observed.

The diagnosis of a hamartoma was confirmed by the histological examination. Microscopically, no cells with 
atypia were found. Fibroblastic-like and epithelioid-like cells admixed with thin collagen fibers could be observed. After 3 months of follow-up, no recurrence was reported.

\section{Discussion}

Breast hamartoma is defined as a well-circumscribed benign lesion composed of glandular tissue, epithelial elements, fibrous tissue and fat, which may be in normal or varying proportions. The term 'breast hamartoma'was first coined by Arrigoni et al in 1971 (1). Affected patients are of all ages, but are mainly adults. A higher predominance of tumors exists in females compared with males. The histological structure of breast hamartoma indicates that the tumor probably results from a dysgenetic factor rather than a tumorous process, as recognized in the World Health Organization in 1981 (6). Although it is histologically benign, in situ and infiltrating carcinomas may arise within hamartomas (7-9). The pathology of hamartoma requires elucidation. The definitive criteria also require identification by pathologists, therefore, the true incidence of this tumor may be underestimated. Fibroadenolipoma, myoid hamartoma and chondrolipoma are histological variations of hamartomas, depending on the proportion of normal breast tissue elements (2). Adipose tissue is commonly reported in the majority of hamartoma cases. The presence of adipose tissue suggests a diagnosis of a hamartoma, but its proportion varies sharply from 5 to $90 \%$ of the lesion volume according to the literature $(10,11)$. Other histological characteristics, including a pseudoangiomatous stroma and epithelial changes, have been described in the literature (12). Although growing slowly, the tumor can develop to a larger size and induce breast asymmetry if excision is not performed in time. Small hamartomas are usually painless and only present as slow-growing breast masses that do not attach to the underlying structure of the breasts. However, large hamartomas may be painful due to compression of the normal breast tissue.

The clinical diagnosis of hamartoma is based on the findings from mammography, sonography and histology examinations. The combination of these methods for the diagnosis is much better than the use of a single method, which may lead to a misdiagnosis. Mammographically, a breast hamartoma is a well-circumscribed mass containing fat and soft tissue. The classic appearance of hamartoma is, at least in part, a mass surrounded by a thin radiopaque line (pseudocapsule), which can often be observed (13). A mammographical diagnosis is made through such findings.

Sonographically, a well-defined mass with an echogenic rim and internal heterogeneity is shown displacing the adjacent normal breast tissue. The presence of a well-circumscribed breast lesion with intact lobular units admixed with various amounts of adipose tissue and a relative paucity of stroma in aspirate form may support the diagnosis of hamartoma pathologically (14). This feature is also useful to differentiate the tumor from a fibroadenoma, with which it may share a clinical and histological resemblance (15).

Lumpectomy is a curative method for hamartoma for which only occasional recurrence has been reported. As a carcinoma may arise within a hamartoma, excision is recommended when the diagnosis is certified.

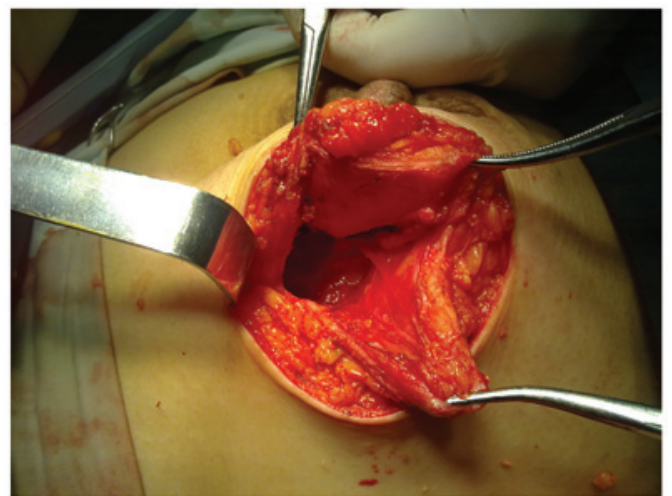

Figure 1. Tumor was located on the upper outer quadrant of the right breast. The surgery was successfully completed and the tumor was completely excised without residual tissue.

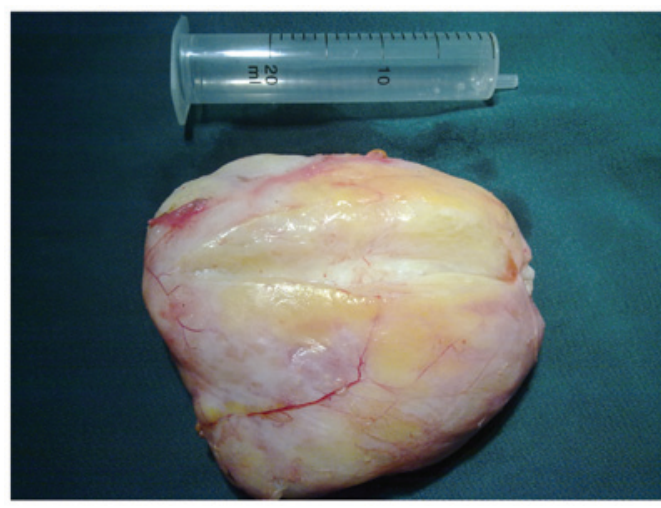

Figure 2. Gross examination revealing a white-reddish, well-defined, encapsulated nodule that was oval, with a smooth margin that could be defined. The nodule was soft and possessed a fibrotic envelope.

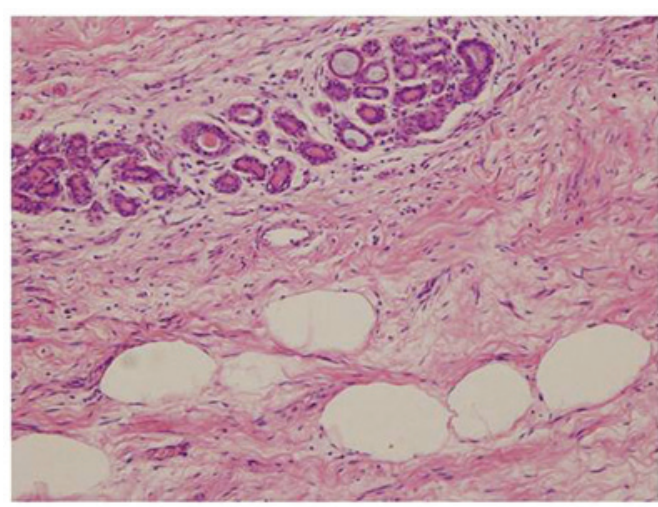

Figure 3. Photomicrograph showing clusters of adipocytes. Adipic area within a fibrotic stroma (haematoxylin and eosin staining; original magnification, $\mathrm{x} 200)$.

In conclusion, a hamartoma is a slow-growing, uncommon breast lesion that possesses certain distinguishing characteristics when mammography, sonography and histology are combined. A definite diagnosis is hard to achieve through a single examination technique. The correlation of imaging and histology findings with the clinical impression is necessary. Although the tumor is histologically benign and often painless, a hamartoma could develop to quite a large size 
if local excision is not performed in time. It should also be realized that although hamartoma are benign, coincidental malignancy may occur. The potential for recurrence is low, but has not been resolved.

\section{References}

1. Arrigoni MG, Dockerty MB and Judd ES: The identification and treatment of mammary hamartomas. Surg Gynecol Obstet 133: 577-582, 1971.

2. Fisher CJ, Hanby AM, Robinson L and Millis RR: Mammary hamartoma - a review of 35 cases. Histopathology 20: 99-106, 1992.

3. Daya D, Trus T, D'Souza TJ, Minuk T and Yemen B: Hamartoma of the breast, an underrecognized breast lesion. A clinicopathologic and radiographic study of 25 cases. Am J Clin Pathol 103: 685-689, 1995.

4. Fechner RE: Fibrodenoma and related lesions. In: Diagnostic Histopathology of the Breast. Page DL and Anderson TJ (eds) Churchill Livingstone, Edinburgh, pp72-85, 1987.

5. Weinzweig N, Botts J and Marcus E: Giant hamartoma of the breast. Plast Reconstr Surg 107: 1216-1220, 2001.

6. World Health Organization: Histologic typing of breast tumor International histological classification of tumors. 2nd edition. Armed Forces Institute of Pathology, Washington, DC, 1991.
7. Scally N, Campbell W, Hall S, McCusker G and Stirling WJ: Invasive ductal carcinoma arising within a breast hamartoma. Ir J Med Sci 180: 767-768, 2011.

8. Kai M, Tada K, Tamura M, Gomi N, Horii R, Akiyama F and Iwase T: Breast cancer associated with mammary hamartoma. Breast Cancer 19: 183-186, 2012.

9. Mester J, Simmons RM, Vazquez MF and Rosenblatt R: In situ and infiltrating ductal carcinoma arising in a breast hamartoma. AJR Am J Roentgenol 175: 64-66, 2000.

10. Jones MW, Norris HJ and Wargotz ES: Hamartomas of the breast. Surg Gynecol Obstet 173: 54-56, 1991.

11. Charpin C, Mathoulin MP, Andrac L, Barberis J, Boulat J, Sarradour B, Bonnier P and Piana L: Reappraisal of breast hamartomas. A morphological study of 41 cases. Pathol Res Pract 190: 362-371, 1994.

12. Herbert M, Schvimer M,Zehavi S, Mendlovic S, Karni T, Pappo I and Sandbank J: Breast hamartoma: Fine-needle aspiration cytologic finding. Cancer 99: 255-258. 2003.

13. Helvie MA, Adler DD, Rebner $\mathrm{M}$ and Oberman HA: Breast hamartomas: Variable mammographic appearance. Radiology 170: 417-421, 1989.

14. Herbert M, Mendlovic S, Liokumovich P, Segal M, Zahavi S, Rath-Wolfson L and Sandbank J: Can hamartoma of the breast be distinguished from fibroadenoma using fine-needle aspiration cytology? Diagn Cytopathol 34: 326-329, 2006.

15. Anani PA and Hessler C: Breast hamartoma with invasive ductcarcinoma. Report of two cases and review of the literature. Pathol Res Pract 192: 1187-1194, 1996. 